\title{
Physikalische Therapie: Die Ambivalenz des Lichts
}

\author{
Jean-Michel Jeannin
}

Basel, Schweiz

\section{Einleitung}

Licht wirkt als natürliches Licht und als Mittel der physikalischen Therapie vielfältig auf den Menschen ein. Licht kann heilen und schädigen. Beides kann von natürlichem und von künstlichem, zur Therapie angewendetem Licht ausgehen. Der Mensch ist natürlicherweise dem Licht ausgesetzt; es ist für ihn lebensnotwendig. Entsprechend wurde früher die Sonne religiös verehrt [1]. Licht kann schwere Hautkrankheiten bis hin zum malignen Melanom auslösen sowie vorbestehende Krankheiten aggravieren. Das Licht, das die Haut trifft, kann dosiert, moduliert und mittels Lichtschutz-Crèmes und -Salben selektiert werden; eine vollständige Expositionsprophylaxe ist jedoch nicht möglich. Einen natürlichen Filter stellt das Ozon dar, das die UV-Einstrahlung auf die Erde reduziert [2].

Der nachfolgende Beitrag befasst sich mit der zuvor beschriebenen Ambivalenz des Lichts, der mit einer Beschreibung seiner Funktionen und Wirkungen beginnt. Im zweiten Abschnitt werden lichtinduzierte Krankheiten und ihre grundsätzlichen Behandlungsmöglichkeiten einschliesslich des Therapiemittels Licht erörtert. Im dritten Teil des Artikels wird eine philosophische Diskussion der Ambivalenz des Lichts versucht.

\section{Biologie des Lichts}

Das natürliche Licht ist für den Menschen lebensnotwendig. Wirksam ist sowohl das sichtbare als auch das unsichtbare (ultraviolette (UV)) Licht. Licht reguliert den Schlaf-Wach-Rhythmus. UV-B-Licht des Wellenbereichs 290-315 nm ist für die Synthese des Vitamin D unerlässlich. Das Mass der Lichtexposition hängt von der geographischen Lage und der Jahreszeit ab. Fensterscheiben sind für den betreffenden Wellenbereich undurchlässig. Die Haut von alten Menschen produziert weniger Vitamin D.
Im Rahmen der Industrialisierung zogen viele Menschen in die Städte um, wo die Lichtexposition unter anderem wegen der Luftverschmutzung und der Bauweise der Wohnhäuser ungenügend war [1]. Eine frühe wissenschaftliche Beschreibung der Folge von Lichtmangel stammt von David Whistler, der in seiner Dissertation aus dem Jahr 1645 die Rachitis («Englische Krankheit») beschreibt [3]. Unter Rachitis wird eine Störung der Knochenbildung und des Knochenwachstums im Kindesalter verstanden [3]. Licht beeinflusst die Stimmung meistens positiv, und Lichtexposition, z.B. am Strand oder im Sonnenstudio, bräunt hellhäutige Menschen und vermittelt Glücksgefühle: Nach UV-A-Exposition fühlten sich die Probanden ausgeglichen, weniger nervös, stärker und mit ihrem Äusseren zufriedener. Zwar wurde eine durch die Haut vermittelte Erhöhung des Serotonins und Melatonins im Blut beobachtet, die aber nach Ansicht der Autoren die beobachteten psychischen Effekte nicht erklären konnten [4]. Lichtempfindliche (photosensible) nicht bildgebende Ganglienzellen der Netzhaut des Auges produzieren Melanopsin, das eine lichtabhängige Unterdrückung der Ausschüttung von Melatonin bewirkt. Melatonin seinerseits wirkt schlafanstossend [5].

\section{Lichtinduzierte Krankheiten}

Es werden akute Reaktionen auf eine zu hohe UV-Bestrahlung von abnormen Reaktionen auf Sonnenlicht unterschieden. Als «Sonnenbrand» wird eine akute, komplexe Hautentzündung nach zu starker Sonnenlicht- oder UV-B-Licht-Exposition bezeichnet. Besonders stark exponiert sind Menschen mit einer sehr hellen Haut. Braune oder gebräunte Haut ist weniger empfindlich. Hauptmerkmal eines Sonnenbrands ist die Rötung. Je nach Schweregrad kommen Schmerzen, Ödeme und Blasen hinzu. In sehr schweren Fällen kann es zu Fieber, Schüttelfrost und Dehydrierung kommen. Zur Behandlung

\section{KARGER}

Fax +497614520714 Information@Karger.com www.karger.com

\section{(c) 2017 S. Karger GmbH, Freiburg}

www.karger.com/szg 
werden mässig wirksame Kortikosteroide empfohlen [6]. Häufiger Sonnenbrand stellt ein Risiko für Hautkarzinome dar $[2,6,7]$. Andauernde, kumulativ hohe Dosen von natürlichem oder künstlichem UV-Licht beschleunigen das Altern der Haut [2] und erhöhen das Risiko für bestimmte Augenkrankheiten, z.B. des Melanoms, der Katarakt und von Bindehauttumoren [7].

Abnorme Reaktionen auf Sonnenlicht äussern sich als Dermatosen (Lichtdermatosen, Photodermatosen). Es werden primäre von sekundären Lichtdermatosen unterschieden. Primäre Lichtdermatosen werden direkt durch die elektromagnetische Strahlung als pathogenetischem Faktor ausgelöst. Sekundären Lichtdermatosen liegt eine meistens internistische Krankheit zugrunde (Lupus erythematodes, Porphyrien, Xeroderma pigmentosum). Die häufigste Photodermatose ist die polymorphe Lichtdermatose (PLD). Sie weist eine Prävalenz von $10-20 \%$ auf (Mitteleuropa, Skandinavien, USA). Kardinalsymptom ist ein stark juckender Hautausschlag. Er kann sich als Flecken, Papeln, Bläschen, Quaddeln oder Plaques äussern, d.h. unter einer Vielzahl von Morphen. Typischerweise tritt die PLD im Frühjahr auf. Die Betroffenen zeigen oft im Verlauf der sonnenreichen Monate einen Gewöhnungseffekt, sodass sie stärkere Sonnenbäder tolerieren. Die Behandlung besteht hauptsächlich in einer Vermeidung der Exposition. Die Prophylaxe geschieht durch Ganzkörperbestrahlung mit UV-A- und/oder UV-B-Licht. Lokal werden Lichtschutzmittel aufgetragen, die ein breites Band von UVStrahlen filtern. Im Unterschied zur PLD ist die Urticaria solaris (Lichturtikaria) eine sehr seltene, aber schwere Lichtdermatose. Sie äussert sich in Quaddeln; in schweren Fällen kann es zum anaphylaktischen Schock kommen. Die Behandlungsmethode der Wahl ist das Auftragen von Psoralen (einer natürlichen lichtsensibilisierenden Substanz) mit konsekutiver Anwendung von UV-A-Strahlen.

Phototoxische Hautreaktionen sind photochemisch ausgelöste Reaktionen ohne immunologische Grundlage im belichteten Bereich der Haut. Wichtige Formen sind die Phytodermatitis (Wiesengräserdermatitis, Dermatitis pratensis) und phototoxische Reaktionen, z.B. durch Tetrazykline. Photosensibilisierende Substanzen können auch endogen, z.B. im Rahmen einer Porphyrie, entstehen. In Verbindung mit photosensibilisierenden Substanzen können UV-Dosen, die normalerweise gut vertragen werden, zu sonnenbrandähnlichen Ausschlägen mit häufiger Hyperpigmentierung führen. Erste therapeutische Massnahme ist das sofortige Absetzen aller phototoxischen Medikamente und Kosmetika (!). Zur Prophylaxe soll ein duftstofffreier Lichtschutz aufgetragen werden. Persistierende Hyperpigmentierungen können mit einer Lasertherapie angegangen werden. Photoallergien sind seltener. Sie treten nur nach vorangegangener Sensibili-

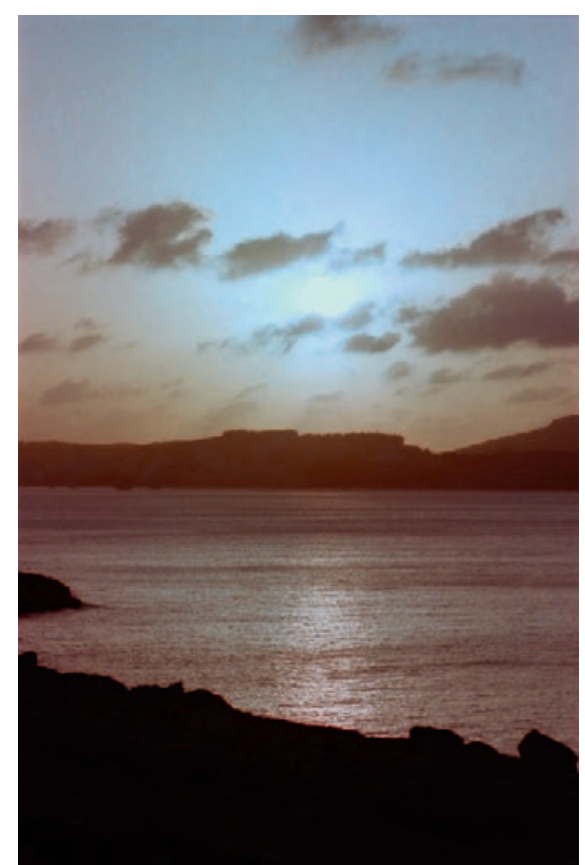

Abb. 1. Die Sonne fasziniert mit ihrem Farbenspiel beim Untergang (Strand auf Malta, 2015).

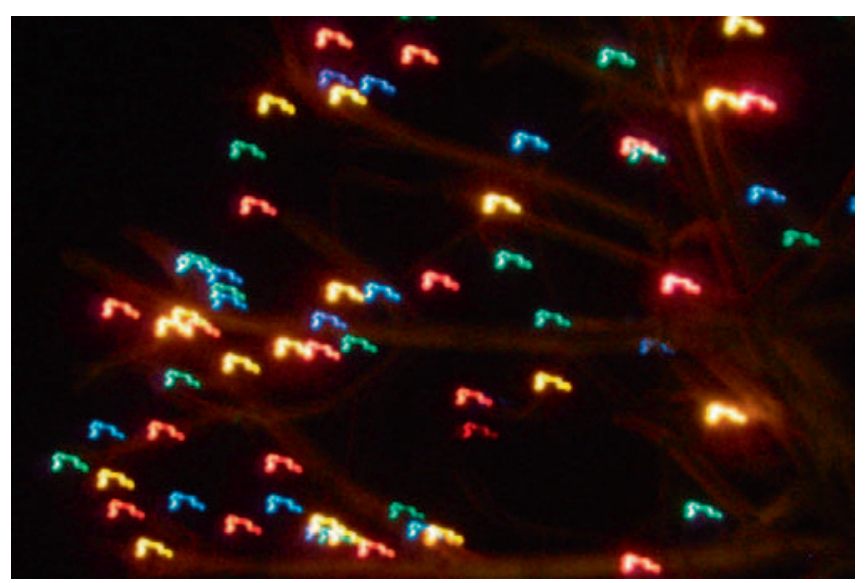

Abb. 2. Farbige Lichter verbreiten in der Dunkelheit der Nacht Heiterkeit (Bad Gastein, 2009).

sierung auf. Als Therapie wird das Photoallergen ausgeschaltet. Zu guter Letzt: Sonnenschutzmittel können selber wiederum unverträglich sein, womit man in einen Circulus vitiosus gelangt [9].

\section{Reflexionen}

Sonnenlicht ist für das Leben des Menschen unabdingbar. Es beeinflusst die Stimmung positiv, ist zur Bildung von Vitamin D unerlässlich, und das Licht reguliert den Schlaf-Wach-Rhythmus (Abb. 1, 2). Lichtmangel macht den Menschen krank. Mit dem physikalischen Therapiemittel Licht in seiner natürlichen oder künstlich erzeugten Form kann eine ganze Reihe von Krankheiten erfolgreich behandelt werden: Depressionen, Schlafstörungen, be- 
stimmte Hautkrankheiten, Gelenkbeschwerden. Laserlicht wird für feinchirurgische Behandlungen verwendet. Andererseits kann Licht auch krank machen. Das gilt für das natürliche Licht wie auch für das künstliche, zu therapeutischen Zwecken hergestellte Licht. Die Doppelnatur des Lichts wird in seinen unerwünschten Wirkungen abgebildet: Die Wellennatur ermöglicht eine Dosierung, ein Zuviel führt zu Hautschäden. Die Materie-Natur setzt das Licht einer Substanz gleich, auf die allergisch reagiert werden kann. Eine weitere Widersprüchlichkeit besteht darin, dass das Licht Krankheiten, die es auslöst, auch zu heilen vermag, z.B. im Rahmen einer Desensibilisierung.

Die Evolution hat die Menschen an die Umgebung, in der sie leben, angepasst. Menschen in sonnenreichen Gegenden sind dunkelhäutig und bekommen keinen Sonnenbrand. Menschen im Norden haben die Pigmentierung der Haut weitgehend verloren; im Extremfall kann sich ihre Haut unter Lichtexposition nicht bräunen. Die weisse pigmentlose Haut nimmt mehr UV-Strahlung auf als die stark pigmentierte, was das Überleben in sonnenarmen Gebieten ermöglicht.

Die hohe Mobilität des Menschen führt dazu, dass er unabhängig von seiner Konstitution z.B. vom Norden in den Süden in die Ferien reisen kann, wo er sich dann vor der Sonne schützen muss - mit Mitteln, auf die er wiederum allergisch reagieren kann. Hier zeigt sich die Ambivalenz des Lichts besonders deutlich.

Die Widersprüchlichkeit des Lichts spiegelt die Evolution wider. Verändert der Mensch langfristig den Ort, wo er lebt, passt sich der Körper an die Änderungen der Ressourcen an. Dass das Licht Störungen, die solche Änderungen auslösen, auch heilen kann, stellt vielleicht einen der Mechanismen (Gewöhnung) dar, der die Evolution antreibt.

Fazit: Es lohnt sich, auf die natürlichen Umstände des Lebens zu achten.

\section{Literatur}

1 Holick MF: Environmental factors that influence the cutaneous production of vitamin D1-3. Am J Clin Nutr 1995;61(suppl):638S645S.

2 Hönigsmann H: Skin diseases in Europe Photodermatology. Eur J Dermatol 2009;19: 658-662.

3 Zhang M, et al: 'English disease': historical notes on rickets, the bone-lung link and child neglect issues. Nutrients 2016:8;pii: E722.
4 Gambichler T, et al: Impact of UVA exposure on psychological parameters and circulating serotonin and melatonin. BMC Dermatology 2002;2:6.

5 Fotosensitive Ganglienzelle. https://de. wikipedia. org/wiki/Fotosensitive_Ganglienzelle (letzter Zugriff: 24.08.2015).

6 Faurschou A, et al: Topical steroids in the treatment of acute sunburn: a randomized, double-blind clinical trial. Arch Dermatol 2008;144:620-624.
7 Gallagher R, et al: Adverse effects of ultraviolet radiation: a brief review. Prog Biophys Mol Biol 2006;92:119-131.

8 Lehmann P, Schwarz T: Lichtdermatosen: Diagnostik und Therapie. Dtsch Arztebl Int 2011;108:135-141.

9 Lim HW, et al: Current challenges in photoprotection. J Am Acad Dermatol 2017;76: S91-S99. 